\title{
System Augmentation and Matrix Updating for Damage Detection in Nonlinear Systems
}

\author{
Kiran D'Souza* and Bogdan Epureanu ${ }^{\dagger}$ \\ Department of Mechanical Engineering \\ University of Michigan \\ 2350 Hayward Street, Ann Arbor, MI 48109-2125
}

\begin{abstract}
A damage detection method is developed for nonlinear systems using model updating. The method uses a nonlinear discrete model of the system and the form of the nonlinearities to create an augmented linear model of the system. A modal analysis technique that uses forcing that is known but not prescribed is then used to solve for the modal properties of the augmented linear system after the onset of damage. Due to the specialized form of the augmentation, the augmented system matrices may not be symmetric, also nonlinear damage causes asymmetrical damage in the updated matrices. A generalized minimum rank perturbation theory is developed to handle the asymmetrical damage scenarios. The damage extent algorithm becomes an iterative process when damage occurs simultaneously in the mass and stiffness matrices. The method is demonstrated using numerical data from two nonlinear mass spring systems and a nonlinear truss. Various damage scenarios of the three nonlinear systems are used to explore the effectiveness of the method. The nonlinearities explored include cubic springs and Coulomb friction.
\end{abstract}

\section{Nomenclature}

A

$\mathbf{A}_{K}$

$\mathbf{A}_{M}$

B

$\mathbf{B}_{K}$

$\mathbf{B}_{M}$

$\mathbf{c}_{i}$

$\mathbf{d}_{i}$

$\mathbf{f}(\mathbf{x}, \dot{\mathbf{x}}, \ddot{\mathbf{x}})$

$\mathbf{g}(t)$

$\mathbf{h}(t)$

$\mathbf{M}, \mathbf{D}, \mathbf{K}$

$\Delta \mathbf{M}, \Delta \mathbf{D}$, $\Delta \mathbf{K}$

$\mathbf{N}_{A I}$

$\mathbf{N}_{A S}$

$\mathbf{N}_{C S}$

$\mathbf{N}_{I}$

$\mathbf{N}_{S}$

$p$

$\mathbf{u}_{d i}$ eigenvectors

eigenvectors using left eigenvectors using right eigenvectors

nonlinear function

external excitation

augmented forcing

and stiffness matrices constants constants constants

rank of the damage damage location matrix using left

force imbalance due to stiffness damage

force imbalance due to mass damage

damage location matrix using right

force imbalance due to stiffness damage

force imbalance due to mass damage

damage location vector of the $i$ th mode

damage location vector of the $i$ th mode

original FEM mass, damping

perturbation to the mass, damping and stiffness matrices due to damage

matrix of nonlinear augmented inertia

matrix of nonlinear augmented stiffness

matrix of nonlinear coupled stiffness

matrix of nonlinear inertia constants

matrix of nonlinear stiffness constants

$i$ th damaged left eigenvector

\footnotetext{
*Email: kdsouza@umich.edu
}

${ }^{\dagger}$ Email: epureanu@umich.edu

$\begin{array}{ll}\mathbf{v}_{d i} & i \text { th damaged right eigenvector } \\ \mathbf{x}(t) & \text { coordinate vector } \\ \mathbf{y}(t) & \text { nonlinear vector } \\ \mathbf{Z}_{d i} & \text { component matrix } \\ \lambda_{d i} & i \text { th damaged eigenvalue } \\ \mathbf{\Omega}_{d} & \text { matrix of damaged natural } \\ & \text { frequencies }\end{array}$

\section{Introduction}

A class of techniques used for structural damage detection is based on vibratory responses, and is focused on identifying changes in linear system behavior. ${ }^{10}$ Such techniques employ linear methods based on matrix updating, and use changes in the natural frequencies and mode shapes. ${ }^{22}$ Other techniques use system identification ${ }^{1,12,19,21,23}$ or generic (and not necessarily physical) models such as neural networks. ${ }^{2,20,26}$

Using modal analysis to obtain the mode shapes and natural frequencies is the first step in many matrix updating approaches. The modal properties are then used in various fashions for damage detection. The linear nondestructive evaluation field has been developed greatly and includes four general categories: optimal matrix updates, sensitivity methods, eigenstructure assignment techniques, and minimum rank perturbation methods (for a review, see review papers by Ibrahim ${ }^{15}$ and Heylen ${ }^{14}$ ).

Many structures in current applications operate in regimes where nonlinear effects are increasingly important. Hence, the field of nonlinear experimental modal analysis is an active area of research which plays an important role in nonlinear vibration-based damage 


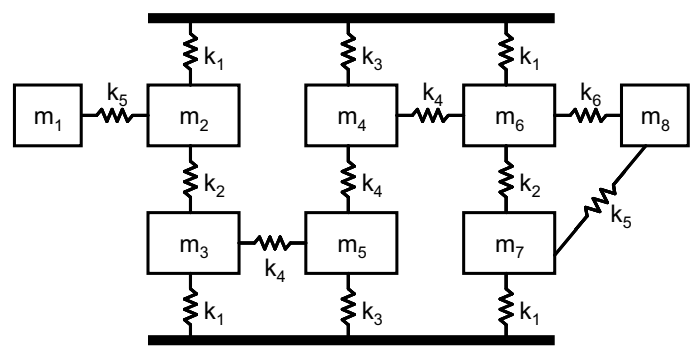

a) Original linear system

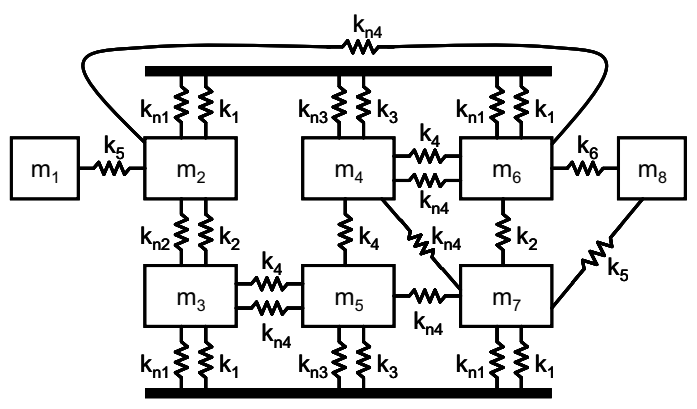

b) Case 1: System with cubic springs

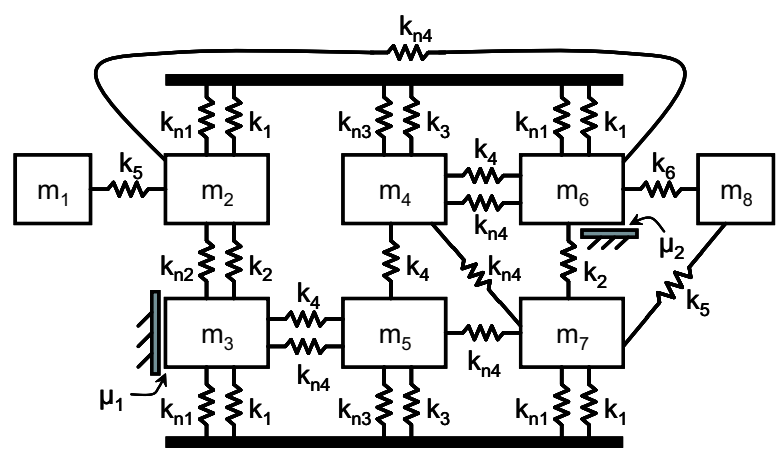

c) Case 2: System with cubic springs \& Coulomb friction

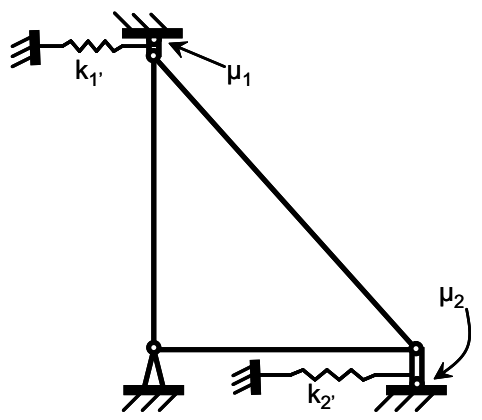

d) Case 3: 2-DOF nonlinear truss

Fig. 1 Systems used to demonstrate the approach

detection. ${ }^{24}$ The field of linear modal analysis is much more developed than its nonlinear counterpart. Linear techniques based on single input single output, single input multiple output, and multiple input multiple output (MIMO) approaches are available. These techniques are developed for both time and frequency domains. Different characteristics of time domain MIMO methods have been discussed by Yang et al. ${ }^{25}$

In this work, an algorithm for analyzing a nonlinear system as an augmented linear system is presented.

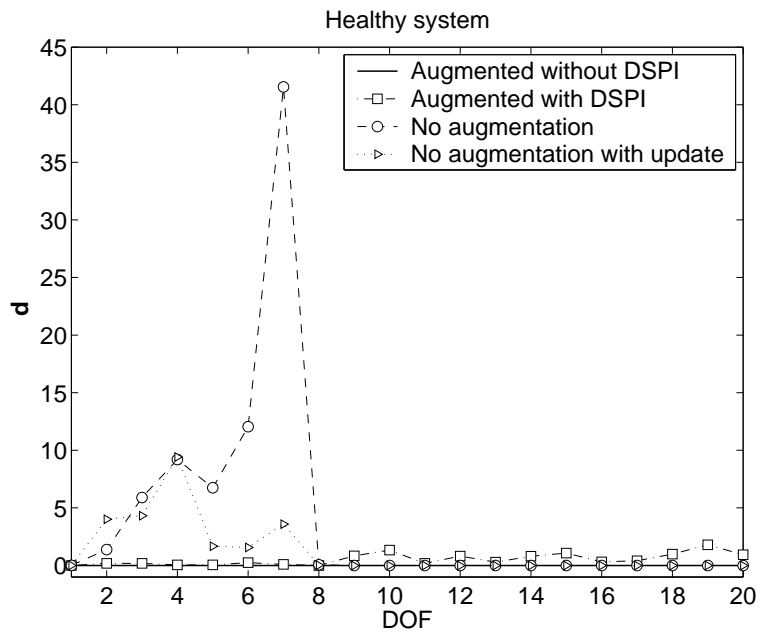

Fig. 2 Case 1: Results of the damage location algorithm applied to a healthy un-augmented nonlinear system and a nonlinear augmented system

This allows for the much better developed areas of linear modal analysis and linear damage detection to be capitalized upon. A key feature of the augmentation requires a modal analysis technique that works with a forcing that is known but not prescribed, such as direct system parameter identification ${ }^{18}$ (DSPI). Next, the modal properties of the augmented system are used in conjunction with a novel generalized minimum rank perturbation theory (GMRPT) to detect the location and extent of damage in nonlinear systems. The proposed GMRPT is designed to account for nonlinearities, and is inspired from a linear damage detection technique employing minimum rank perturbation theory (MRPT). ${ }^{17,27-30}$

To demonstrate the proposed approach, the method is applied to three nonlinear systems. The effectiveness of the augmentation and the GMRPT are demonstrated by numerical experiments. Also, the influence of inaccuracies in modal parameters is analyzed.

In the following, the procedure for detecting the location and extent of damage in nonlinear systems using linear theories is demonstrated. First, the modeling of the nonlinear system by an augmented linear one is introduced. Then, a means to solve the eigenvalue problem for the augmented system using DSPI ${ }^{18}$ is detailed. Finally, the equations for GMRPT, which are used for damage detection, are presented.

In addition to the general theory, the technique is applied to three nonlinear systems, shown in parts (b), (c) and (d) of Fig. 1. The first two nonlinear systems, shown in parts (b) and (c) of Fig. 1, are based on an eight degree of freedom (DOF) mass-spring system (Kabe system ${ }^{27-30}$ ) shown in part (a) of Fig. 1. Case 1, shown in part (b) of Fig. 1, is the linear Kabe system expanded with twelve nonlinear springs of the form $k_{n} \Delta x^{3}$, where $k_{n}$ is the nonlinear spring stiffness, and $\Delta x$ represents the distance the nonlinear spring is 


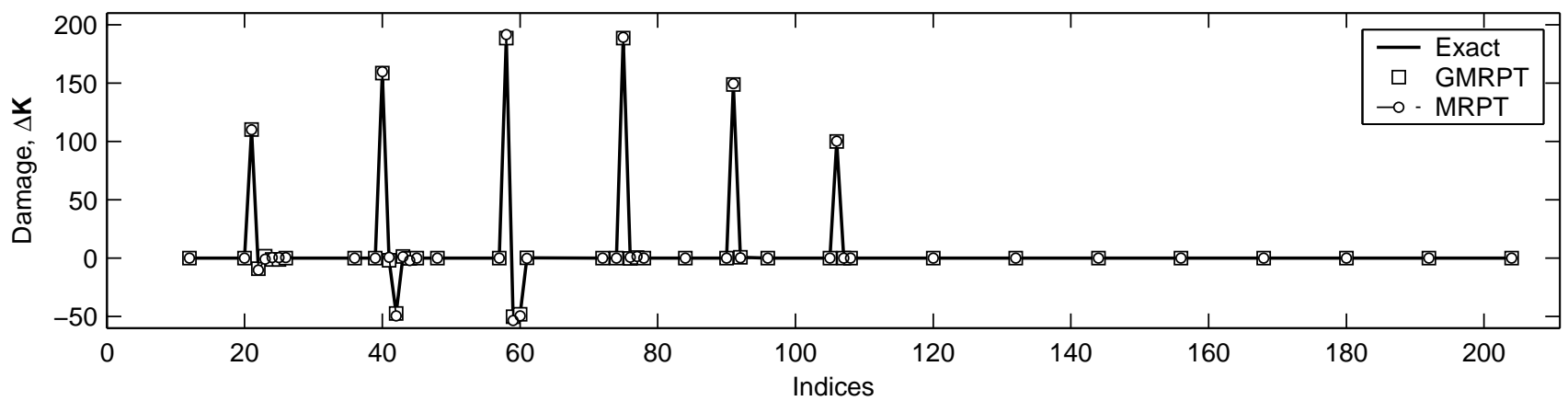

a) Linear spring damage

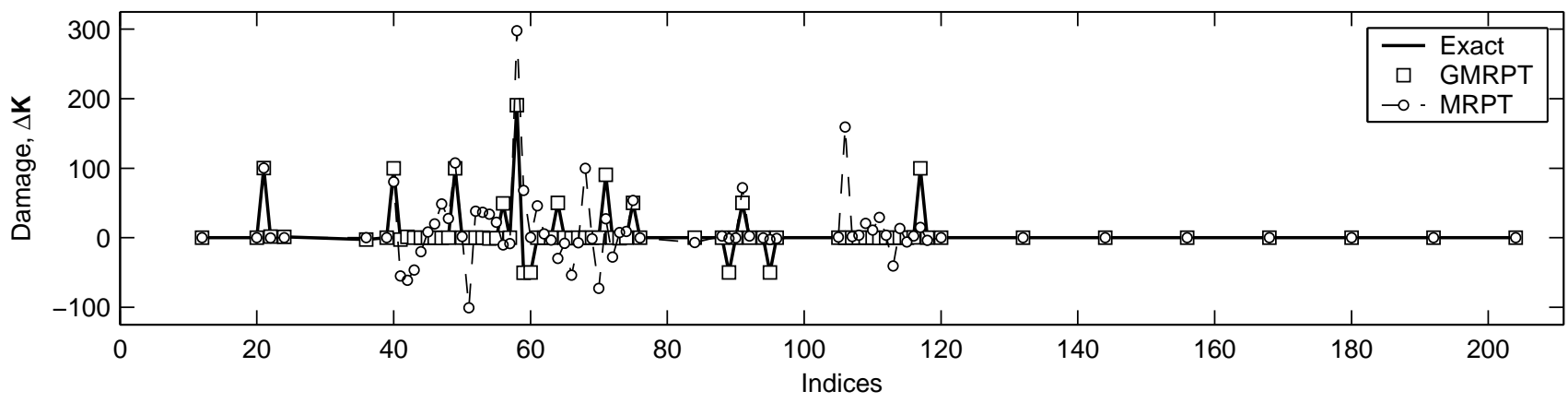

b) Nonlinear and linear spring damage

Fig. 3 Case 1: A comparison of results obtained using GMRPT and MRPT for simultaneous damage in ten linear springs (a) and five linear and five nonlinear springs (b)

stretched. Case 2, shown in part (c) of Fig. 1, is the same as case 1 with the addition of Coulomb friction of the form $\mu|\dot{x}|$ at masses three and six. Case 3, shown in part (d) of Fig. 1, is a two degree of freedom truss with each of the pinned joints having Coulomb friction, and connected to ground by a cubic spring.

\section{System Augmentation for Modeling Nonlinear Systems}

In this section, a method to model a nonlinear system by an augmented linear system is presented. Consider a nonlinear system characterized by a coordinate vector $\mathbf{x}$ and forced by an external excitation $\mathbf{g}(t)$, and expressed as

$$
\begin{aligned}
\mathbf{M} \ddot{\mathbf{x}}+\mathbf{D} \dot{\mathbf{x}}+\mathbf{K} \mathbf{x}+\mathbf{f}(\mathbf{x}, \dot{\mathbf{x}}, \ddot{\mathbf{x}}) & =\mathbf{g}(t), \text { or } \\
{\left[\begin{array}{cc}
\mathbf{M} & \mathbf{0} \\
\mathbf{0} & \mathbf{I}
\end{array}\right]\left[\begin{array}{c}
\ddot{\mathbf{x}} \\
\dot{\mathbf{x}}
\end{array}\right]+\left[\begin{array}{cc}
\mathbf{D} & \mathbf{K} \\
-\mathbf{I} & \mathbf{0}
\end{array}\right]\left[\begin{array}{c}
\dot{\mathbf{x}} \\
\mathbf{x}
\end{array}\right] } & + \\
{\left[\begin{array}{c}
\mathbf{f}(\mathbf{x}, \dot{\mathbf{x}}, \ddot{\mathbf{x}}) \\
\mathbf{0}
\end{array}\right] } & =\left[\begin{array}{c}
\mathbf{g}(t) \\
\mathbf{0}
\end{array}\right],
\end{aligned}
$$

where $\mathbf{M}, \mathbf{D}$, and $\mathbf{K}$ are the mass, damping and stiffness matrices, and $\mathbf{f}$ is a nonlinear function. For certain forms of nonlinearities, Eq. (1) can be rewritten as

$$
\begin{gathered}
\mathbf{M} \ddot{\mathbf{x}}+\mathbf{D} \dot{\mathbf{x}}+\mathbf{K} \mathbf{x}+\mathbf{N}_{I} \ddot{\mathbf{y}}+\mathbf{N}_{S} \mathbf{y}=\mathbf{g}(t), \quad \text { or } \\
{\left[\begin{array}{ccc}
\mathbf{M} & \mathbf{0} & \mathbf{N}_{I} \\
\mathbf{0} & \mathbf{I} & \mathbf{0} \\
\mathbf{0} & \mathbf{0} & \mathbf{N}_{A I}
\end{array}\right]\left[\begin{array}{c}
\ddot{\mathbf{x}} \\
\dot{\mathbf{x}} \\
\ddot{\mathbf{y}}
\end{array}\right]+}
\end{gathered}
$$

$$
\left[\begin{array}{ccc}
\mathbf{D} & \mathbf{K} & \mathbf{N}_{S} \\
-\mathbf{I} & \mathbf{0} & \mathbf{0} \\
\mathbf{0} & \mathbf{N}_{C S} & \mathbf{N}_{A S}
\end{array}\right]\left[\begin{array}{c}
\dot{\mathbf{x}} \\
\mathbf{x} \\
\mathbf{y}
\end{array}\right]=\left[\begin{array}{c}
\mathbf{g}(t) \\
\mathbf{0} \\
\mathbf{h}(t)
\end{array}\right]
$$

where $\mathbf{N}_{I}, \mathbf{N}_{A I}, \mathbf{N}_{S}, \mathbf{N}_{C S}$ and $\mathbf{N}_{A S}$ are constant matrices (more details are presented in the next paragraphs), and $\mathbf{y}$ contains nonlinear terms. The function $\mathbf{h}(t)$ in Eq. (2) is introduced to preserve most of the properties of the matrices in Eq. (1). The augmentation is expressed such that it matches the form chosen for the nonlinear systems used in this paper. However, the system can be augmented differently to optimally suit various applications. Eq. (2) is the augmented linear model of the nonlinear system for which the eigenvalue problem must be solved.

To demonstrate the proposed approach, we employ two nonlinear Kabe systems and a nonlinear truss shown in Fig. 1. The nonlinear springs are considered to have cubic nonlinearity, and their stiffnesses are denoted by an $n$ in the subscript (i.e. $k_{n i}$ ). The location of the Coulomb friction is denoted by $\mu_{i}$.

For the types of nonlinearities explored in this paper, the augmentation was chosen such that the constants in $\mathbf{N}_{S}$ correspond to the parameters $k_{n i}$, and they multiply y. For example, for one cubic spring the corresponding augmented entry in $\mathbf{y}$ is $\Delta x_{i j}^{3}$. The constants in $\mathbf{N}_{I}$ correspond to $\mu_{i}$, and they multiply a term of the form $\ddot{\mathbf{y}}$. For Coulomb friction the corresponding augmented entry in $\ddot{\mathbf{y}}$ is $\left|\dot{\mathbf{x}}_{i}\right|$. The augmentations were 


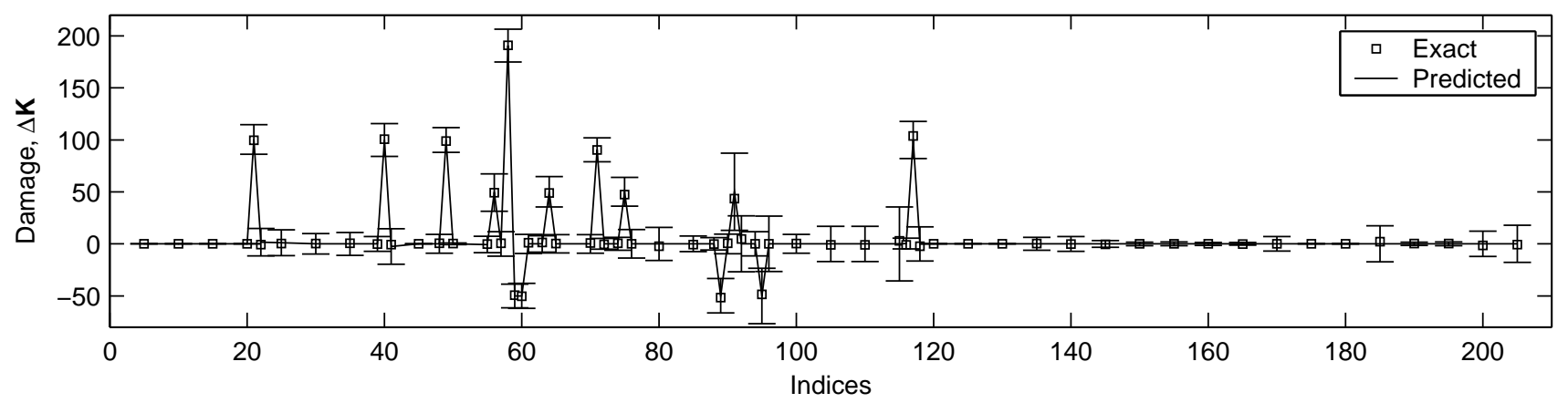

Fig. 4 Case 1: Predicted damage in the nonlinear Kabe system with simultaneous damage in five linear and five nonlinear springs for a case with $1 \%$ random eigenvector noise and \pm 0.25 random eigenvalue noise

chosen in this manner to keep the constant matrices $\mathbf{N}_{S}$ and $\mathbf{N}_{I}$ to the same order of magnitude as the linear matrices $\mathbf{K}$ and $\mathbf{M}$, respectively. Also, an advantage of having the Coulomb friction in the mass matrix is that $\left|\dot{\mathbf{x}}_{i}\right|$ does not have to be differentiated in order to obtain $\mathbf{h}(t)$, rather it can be integrated, and that alleviates problems associated with the nonsmoothness caused by Coulomb friction.

The following is an example of how the augmentation is applied for a one degree of freedom system with a mass connected to ground by a linear and nonlinear spring. The equation of motion of this nonlinear system can be written as

$$
m \ddot{x}+k x+k_{n} x^{3}=g(t),
$$

where $m$ is the mass, $k$ is the linear spring stiffness, and $k_{n}$ is the nonlinear spring stiffness. Using the augmentation described in this paper the new augmented system would be represented by the following

$$
\begin{aligned}
m \ddot{x}+k x+N_{S} y & =g(t), \\
N_{A I} \ddot{y}+N_{C S} x+N_{A S} y & =h(t) .
\end{aligned}
$$

In this case $N_{S}$ and $N_{C S}$ are simply $k_{n}$ and $y$ is $x^{3}$, while $N_{A I}$ and $N_{A S}$ are constants of our choosing. In this paper values of order of the mass matrix were chosen for $N_{C S}$, and $2 k_{n}$ was chosen for $N_{A S}$.

The augmentation for Coulomb friction is slightly different. The following is an example of how the augmentation is applied to a one degree of freedom mass connected to the ground by a linear spring and also rubbing against the ground. The equation of motion of this nonlinear system can be written as

$$
m \ddot{x}+k x+\mu|\dot{x}|=g(t),
$$

where $\mu$ is the coefficient of friction for Coulomb friction. Using the augmentation described in this paper the new augmented system would be represented by the following

$$
\begin{aligned}
m \ddot{x}+k x+N_{I} \ddot{y} & =g(t), \\
N_{A I} \ddot{y}+N_{C S} x+N_{A S} y & =h(t) .
\end{aligned}
$$

In this case $\ddot{y}$ is $|\dot{x}|$ and $N_{I}$ is $\mu$, while $N_{A I}, N_{C S}$ and $N_{A S}$ are constants of our choosing. In this paper $\mu$ was chosen for $N_{A I}$, and a constant was chosen for $N_{A S}$, while $N_{C S}$ was simply the opposite of $N_{A S}$.

To use the augmented model, a modal analysis technique which uses an excitation that is known, but not prescribed (such as DSPI) is needed. DSPI enables one to determine the mode shapes and natural frequencies of the system when the displacement of the degrees of freedom $(\mathbf{x}(t)$ and $\mathbf{y}(t))$ and the forcing $(\mathbf{f}(t)$ and $\mathbf{h}(t))$ are known.

An example of implementation of the proposed approach is to measure the displacement vector $\mathbf{x}(t)$ and the forcing vector $\mathbf{f}(t)$. The vector $\mathbf{y}(t)$ may then be computed from $\mathbf{x}(t)$, and the vector $\mathbf{h}(t)$ may be calculated to satisfy Eq. (2). The requirement of the modal analysis technique to use a known but not prescribed forcing streams from the known forcing $\mathbf{h}(t)$.

A consequence of the form of the augmentation is the inability for damage to be modeled in the augmentation. This means that damage occurring in $\mathbf{N}_{S}$ or $\mathbf{N}_{I}$ will only be reflected in the linear portion, and not the augmented portion of the system. The end result is that nonlinear damage causes asymmetrical changes in the system matrices when using this augmentation.

\section{Generalized Minimum Rank Perturbation Theory}

In this section the primary equations of the proposed generalized MRPT are stated. The proof is presented in a longer paper. ${ }^{7}$ The proposed GMRPT technique is specifically designed for the cases with asymmetric damage in the augmented system. The derivation of these formulas is omitted in this paper for the sake of brevity, the equations follow closely MRPT. ${ }^{17,28}$ The damage location is identified based on the following equation

$$
\mathbf{d}_{i} \equiv \mathbf{Z}_{d i} \mathbf{v}_{d i}=\left(\lambda_{d i}^{2} \Delta \mathbf{M}+\lambda_{d i} \Delta \mathbf{D}+\Delta \mathbf{K}\right) \mathbf{v}_{d i},
$$

where $\mathbf{Z}_{d i} \equiv \lambda_{d i}^{2} \mathbf{M}+\lambda_{d i} \mathbf{D}+\mathbf{K}, \lambda_{d i}, \mathbf{v}_{d i}$, and $\mathbf{u}_{d i}$ are the $i$ th eigenvalue, right eigenvector and left eigenvector. 


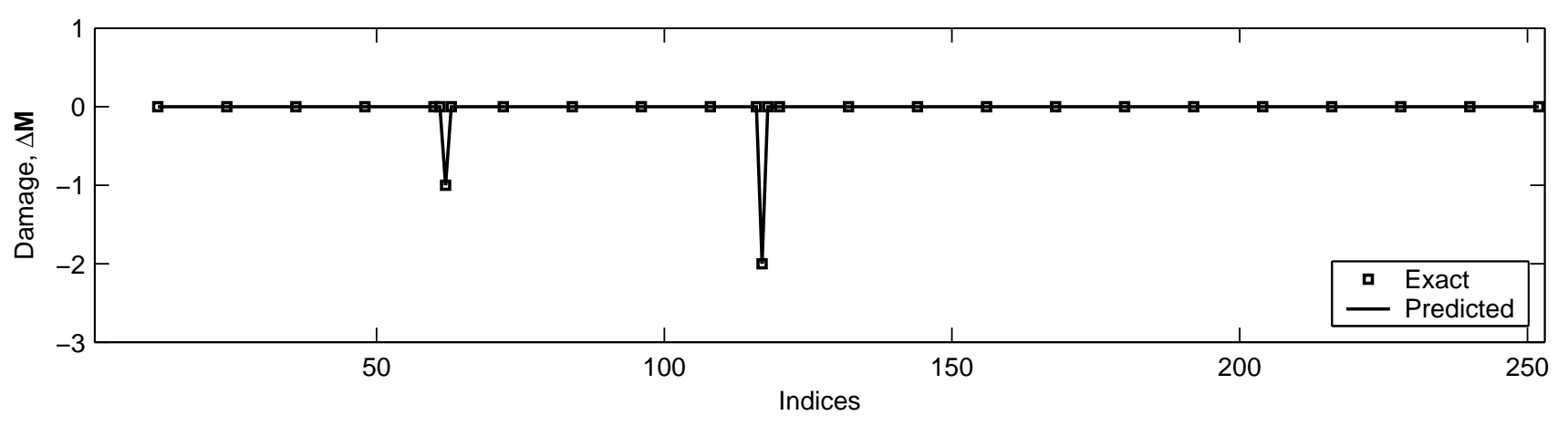

a) Damage in the mass matrix

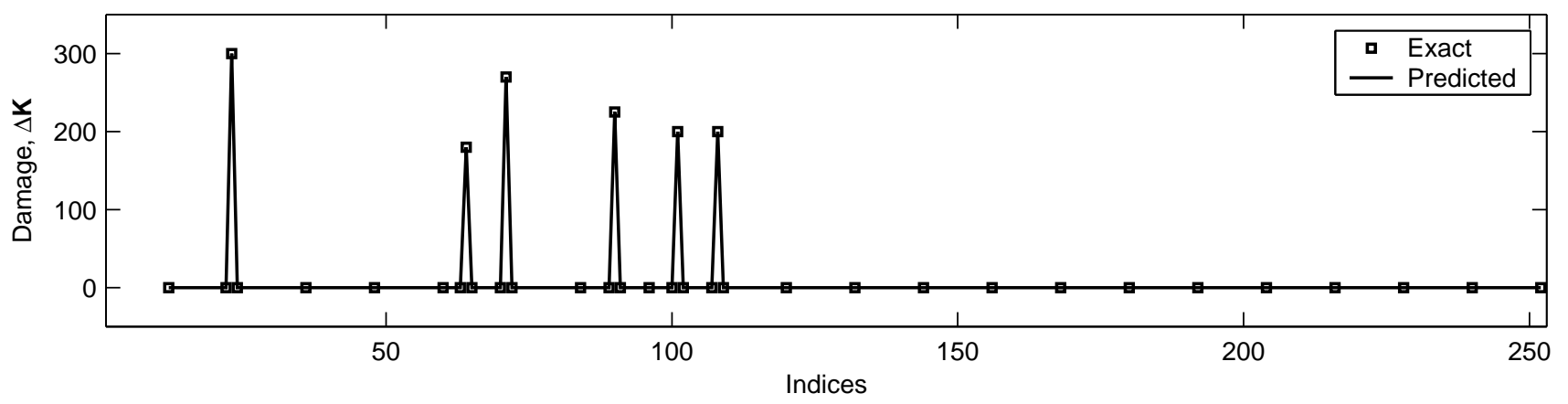

b) Damage in the stiffness matrix

Fig. 5 Case 2: Predicted damage in the nonlinear Kabe system with damage associated with an increase in Coulomb friction (a), and a reduction of stiffness in three linear and three nonlinear springs (b) for a case with exact eigenvalues and right eigenvectors

A composite damage vector may be defined from the multiple measured modes as

$$
\mathbf{d}=\frac{1}{p} \sum_{i=1}^{p} \frac{\mathbf{d}_{i}}{\left\|\mathbf{v}_{d i}\right\|}
$$

For identifying the extent of the damage, for this paper it is assumed that there is no proportional damping. Hence, the changes caused by damage are in the stiffness matrix, $\Delta \mathbf{K}$, and mass mass matrix, $\Delta \mathbf{M}$. One can define the following perturbation matrices

$$
\begin{aligned}
\mathbf{B}_{M} & =\mathbf{B}\left(\mathbf{U}_{d}^{T} \mathbf{B}\right)^{-1}\left(\mathbf{U}_{d}^{T} \mathbf{M} \mathbf{V}_{d}-\mathbf{I}_{p \times p}\right), \\
\mathbf{B}_{K} & =\mathbf{B}\left(\mathbf{U}_{d}^{T} \mathbf{B}\right)^{-1}\left(\mathbf{U}_{d}^{T} \mathbf{K} \mathbf{V}_{d}-\boldsymbol{\Omega}_{d}\right), \\
\mathbf{A}_{M} & =\mathbf{A}\left(\mathbf{V}_{d}^{T} \mathbf{A}\right)^{-1}\left(\mathbf{V}_{d}^{T} \mathbf{M}^{T} \mathbf{U}_{d}-\mathbf{I}_{p \times p}\right), \\
\mathbf{A}_{K} & =\mathbf{A}\left(\mathbf{V}_{d}^{T} \mathbf{A}\right)^{-1}\left(\mathbf{V}_{d}^{T} \mathbf{K}^{T} \mathbf{U}_{d}-\boldsymbol{\Omega}_{d}\right),
\end{aligned}
$$

where $\mathbf{I}_{p \times p}$ is the identity matrix and

$$
\begin{aligned}
\mathbf{V}_{d} & =\left[\mathbf{v}_{d 1}, \mathbf{v}_{d 2}, \ldots, \mathbf{v}_{d p}\right], \\
\mathbf{U}_{d}^{T} & =\left[\mathbf{u}_{d 1}, \mathbf{u}_{d 2}, \ldots, \mathbf{u}_{d p}\right]^{T}, \\
\mathbf{B} & =\left[\mathbf{d}_{1}, \mathbf{d}_{2}, \ldots, \mathbf{d}_{p}\right], \\
\mathbf{A}^{T} & =\left[\mathbf{c}_{1}, \mathbf{c}_{2}, \ldots, \mathbf{c}_{p}\right]^{T}, \\
\mathbf{c}_{i}^{T} & \equiv \mathbf{u}_{d i}^{T} \mathbf{Z}_{d i}=\mathbf{u}_{d i}^{T}\left(\lambda_{d i}^{2} \Delta \mathbf{M}+\lambda_{d i} \Delta \mathbf{D}+\Delta \mathbf{K}\right) .
\end{aligned}
$$

The singular value decomposition of the matrices defined in Eq. (8) yields

$$
\mathbf{B}_{M}=\mathbf{U}_{B M} \mathbf{S}_{B M} \mathbf{V}_{B M}^{T},
$$

$$
\begin{aligned}
\mathbf{B}_{K} & =\mathbf{U}_{B K} \mathbf{S}_{B K} \mathbf{V}_{B K}^{T}, \\
\mathbf{A}_{M} & =\mathbf{U}_{A M} \mathbf{S}_{A M} \mathbf{V}_{A M}^{T}, \\
\mathbf{A}_{K} & =\mathbf{U}_{A K} \mathbf{S}_{A K} \mathbf{V}_{A K}^{T},
\end{aligned}
$$

whereby one can define

$$
\begin{aligned}
\mathbf{Z}_{M} & =\mathbf{V}_{B M} \mathbf{S}_{B M}, \\
\mathbf{Z}_{K} & =\mathbf{V}_{B K} \mathbf{S}_{B K}, \\
\mathbf{Y}_{M} & =\mathbf{V}_{A M} \mathbf{S}_{A M}, \\
\mathbf{Y}_{K} & =\mathbf{V}_{A K} \mathbf{S}_{A K} .
\end{aligned}
$$

Using the matrices defined in Eq. (8) and Eq. (10) the perturbation to the mass and stiffness matrices can be expressed as

$$
\begin{aligned}
\Delta \mathbf{M} & =\mathbf{B}_{M} \mathbf{Z}_{M}\left(\mathbf{Y}_{M}^{T} \mathbf{A}_{M}^{T} \mathbf{V}_{d} \mathbf{Z}_{M}\right)^{-1} \mathbf{Y}_{M}^{T} \mathbf{A}_{M}^{T}, \\
\Delta \mathbf{K} & =\mathbf{B}_{K} \mathbf{Z}_{K}\left(\mathbf{Y}_{K}^{T} \mathbf{A}_{K}^{T} \mathbf{V}_{d} \mathbf{Z}_{K}\right)^{-1} \mathbf{Y}_{K}^{T} \mathbf{A}_{K}^{T} .
\end{aligned}
$$

To identify the damage location, only right eigenvectors are needed in GMRPT. However, to identify the damage extent using GMRPT, $p$ of the $n$ left eigenvectors of the system are needed as well. One way of obtaining the left eigenvectors from the right eigenvectors is to use the mass orthogonality property of the eigenvectors given by

$$
\begin{aligned}
& \mathbf{U}_{d}^{T} \mathbf{M}_{d} \mathbf{V}_{d}=\mathbf{I}, \quad \text { so } \mathbf{U}_{d}^{T}=\mathbf{V}_{d}^{-1} \mathbf{M}_{d}^{-1} \\
& \mathbf{U}_{d}^{T} \mathbf{K}_{d} \mathbf{V}_{d}=\boldsymbol{\Omega}_{d}, \text { so } \mathbf{U}_{d}^{T}=\boldsymbol{\Omega}_{d} \mathbf{V}_{d}^{-1} \mathbf{K}_{d}^{-1} .
\end{aligned}
$$




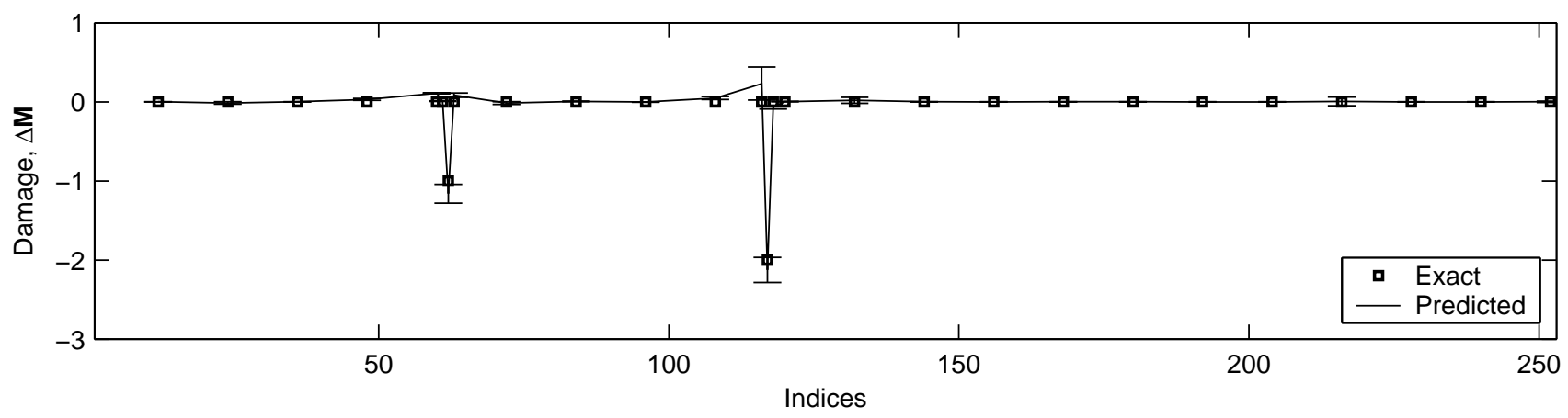

a) Damage in the mass matrix

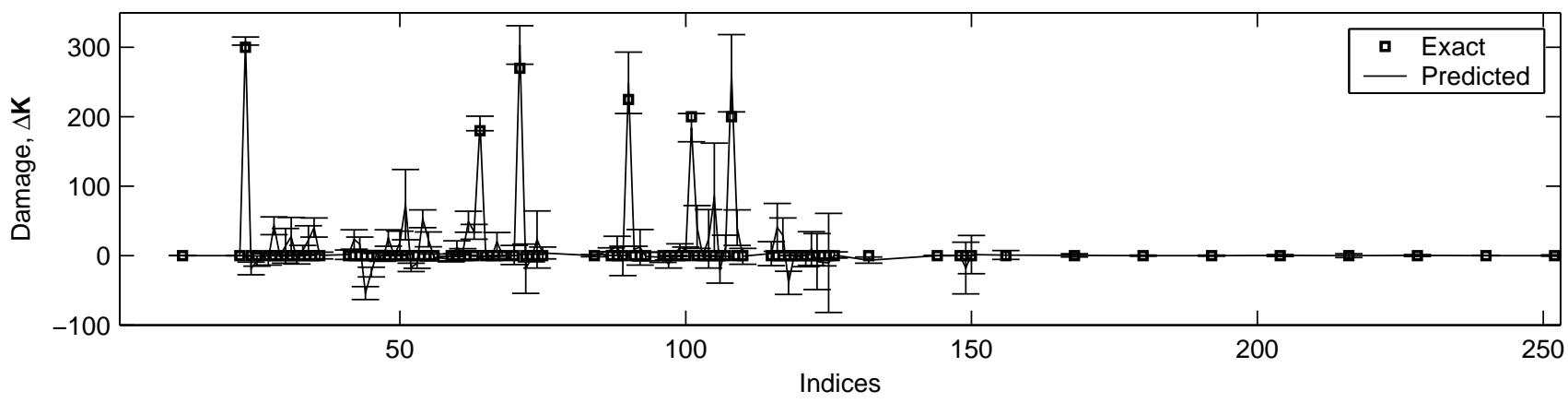

b) Damage in the stiffness matrix

Fig. 6 Case 2: Predicted damage in the nonlinear Kabe system with damage associated with an increase in Coulomb friction (a), and a reduction of stiffness in three linear and three nonlinear springs (b) for a case with $3 \%$ random eigenvector noise and $1 \%$ random eigenvalue noise

An approach based on Eq. (12) and Eq. (13) requires the knowledge of all $n$ right eigenvectors of the system to be known in order to solve for the left ones. In many vibratory problems in structural dynamics and/or fluid-structure interactions, the system of interest is modeled accurately by a system of equations of motion which is large dimensional (i.e. $n$ is very large). Recent techniques for reducing the complexity of these models employ reduced order modeling ${ }^{3-6,8,9,11,13,16}$ based on approaches such as component mode synthesis and proper orthogonal decomposition. These techniques are applicable to both linear and nonlinear systems and usually provide a transformation from the high-dimensional space of displacements $\mathbf{x}$ (of size $n$ ) to a reduced order space $\mathbf{q}$ (of size $r<<n$ ) as $\mathbf{x}=\mathbf{P q}$, where $\mathbf{P}$ is a $n \times r$ matrix. Next, the equations of motion are expressed in the reduced order space. For example, Eq. (1) successively becomes

$$
\begin{aligned}
\mathbf{M P} \ddot{\mathbf{q}}+\mathbf{D P} \dot{\mathbf{q}}+\mathbf{K P q} & =\mathbf{0} \\
\mathbf{P}^{T} \mathbf{M P} \ddot{\mathbf{q}}+\mathbf{P}^{T} \mathbf{D P} \dot{\mathbf{q}}+\mathbf{P}^{T} \mathbf{K} \mathbf{P q} & =\mathbf{0} \\
\overline{\mathbf{M}} \ddot{\mathbf{q}}+\overline{\mathbf{D}} \dot{\mathbf{q}}+\overline{\mathbf{K}} \mathbf{q} & =\mathbf{0} .
\end{aligned}
$$

The reduced order equation of motion in Eq. (14) has a low order $r$. Hence, its $r$ right eigenvectors may be measured much more easily than the $n$ right eigenvectors of the original problem. Once the $r$ reduced order right eigenvectors are obtained, the $r$ left eigen- vectors $\mathbf{e}_{i}$ (for $i=1, \ldots, r$ ) of the reduced order model may be computed using a relation similar to Eq. (12) and Eq. (13) by using the reduced order mass matrix $\overline{\mathbf{M}}$. Next, the $r$ most dominant full size left eigenvectors are obtained as $\mathbf{u}_{d i}=\mathbf{P} \mathbf{e}_{i}$, for $i=1, \ldots, r$. This method is contingent upon using the lowest frequencies of the system to do the reduced order modeling. In practice it is found that higher frequencies are more sensitive to damage. The GMRPT is not restricted to using the lowest frequency modes as long as the left and right eigenvectors are known (in the frequency range where damage manifests primarily).

\section{Numerical Results}

To demonstrate the proposed methodology, a numerical analysis of the nonlinear systems in Fig. 1 was performed. The matrices $\mathbf{M}, \mathbf{K}, \mathbf{N}_{I}$ and $\mathbf{N}_{S}$ were obtained for the selected system, and each mass was forced harmonically. The vector of displacements $\mathbf{x}(t)$ was calculated by standard time integration, while $\mathbf{y}(t)$ and $\mathbf{h}(t)$ were calculated based on their relation to $\mathbf{x}(t)$. DSPI was employed for the augmented system to determine the eigenvalues and eigenvectors of the augmented matrices by using the time series for $\mathbf{x}(t), \mathbf{y}(t)$, $\mathbf{f}(t)$, and $\mathbf{h}(t)$. Next, GMRPT was used to determine the damage location and extent by using the modal data provided by DSPI. Various numerical simulations were run to demonstrate the effectiveness of the augmentation, the benefits of GMRPT over MRPT, and 


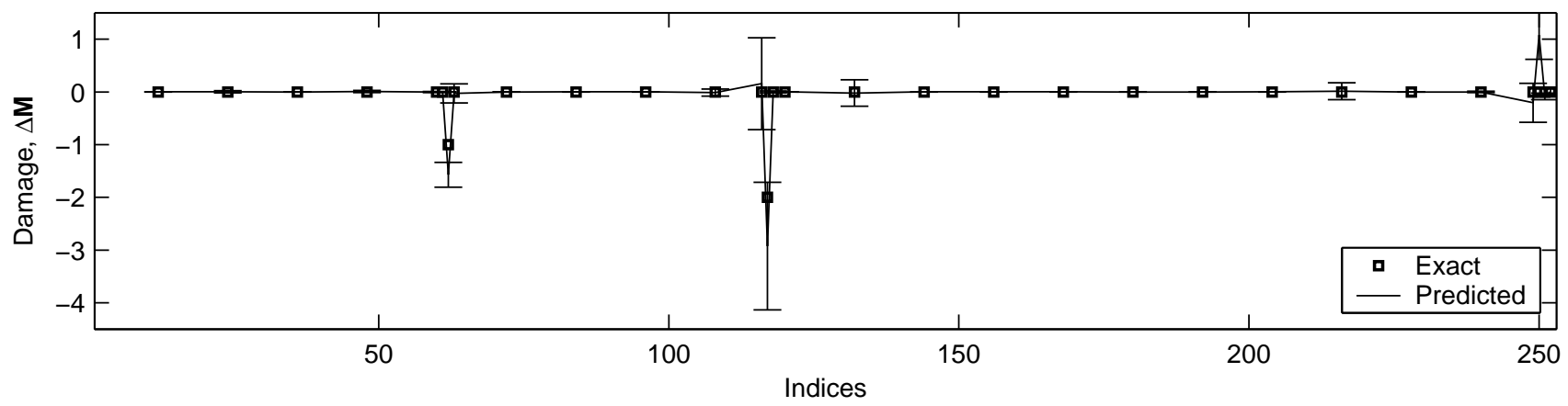

a) Damage in the mass matrix

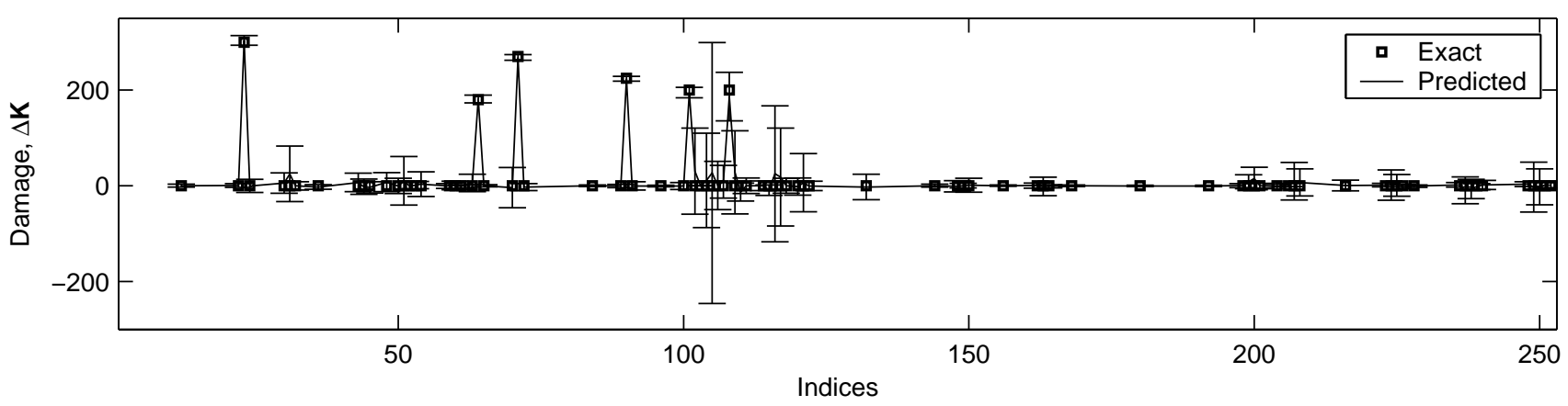

b) Damage in the stiffness matrix

Fig. 7 Case 2: Predicted damage in the nonlinear Kabe system with damage associated with an increase in Coulomb friction (a), and a reduction of stiffness in three linear and three nonlinear springs (b) for a case with $0.01 \%$ random noise input into DSPI

the effect of random noise.

\section{Case 1: Kabe System with Cubic Springs}

The effect of nonlinearities on the healthy system can be seen in Fig. 2. The plot shows the damage location obtained using Eq. (7) for four different cases. The first case represents an augmented system and a calculation where the exact eigenvalues and eigenvectors are used. This accurately predicts that no damage is present in any of the degrees of freedom. The second case represents an augmented system and a calculation where DSPI is used to obtain eigenvalues and eigenvectors. These results show a very good estimate, with slight deviations predicted by GMRPT in the augmented degrees of freedom due to small inaccuracies in solving the eigenvalue problem by DSPI. In contrast, the third case uses DSPI to obtain the eigenvalues and eigenvectors also, but the system is modeled as the original linear system even though there are twelve nonlinear springs. The results show that damage is erroneously predicted by MRPT in all the linear DOF, except one and eight.

The erroneous predictions of the linear MRPT are not alleviated by matrix updating. To show that, the stiffness matrix was updated with the damage predicted using MRPT, and the forcing $\mathbf{f}(t)$ was slightly altered to simulate a change in forcing with time. The results are shown in Fig. 2 where the last case used DSPI with the updated linear model. Although the erroneous damage decreases, a significant amount of damage resides after model updating. This updated case shows that the linear model is inaccurate when used to model a nonlinear system, despite matrix updating because damage is still erroneously predicted at the degrees of freedom that contain nonlinearities.

For the case where the damage to a system is solely in its linear components, GMRPT predicts the same damage location and extent as MRPT. Fig. 3 presents element by element the values of the stiffness perturbation matrices $(\Delta \mathbf{K})$ obtained using MRPT and separately GMRPT (and using exact eigenvalues and eigenvectors of the augmented system). The $x$-axes in each plot represent the index of a column vector obtained from storing the upper triangular portion of the perturbation stiffness matrix $(\Delta \mathbf{K})$ into a column vector. The $y$-axes in the plots represent the entries of the difference between the original and updated stiffness matrices, $\Delta \mathbf{K}$. Part (a) of Fig. 3 is the case where ten linear springs are damaged. Since the damage is linear, both MRPT and GMRPT predict accurately the exact damage in all ten linear springs. Part (b) of Fig. 3 is the case where five linear springs and five nonlinear springs are damaged. GMRPT is able to predict accurately the exact damage in both linear and nonlinear springs, while MRPT incorrectly predicts the damage in the nonlinear springs.

To determine the sensitivity of the proposed method to measurement noise, a $1 \%$ random eigenvector per- 
turbation, and a \pm 0.25 random eigenvalue perturbation was added to the case where five linear and five nonlinear springs were damaged. The results are illustrated in Fig. 4. The predicted damage is shown in a similar fashion as in Fig. 3. The average damage values for 100 separate calculations were obtained for each damage index, and standard deviation error bars are plotted. The figure shows that the average value of the predicted damage is very close to the exact damage (e.g. within $1 \%$ of the exact damage values).

\section{Case 2: Kabe System with Cubic Springs and Coulomb Friction}

This case expands the types of nonlinearities the novel GMRPT and augmentation method are used for by simultaneously including cubic springs and Coulomb friction for the mass spring system. In addition this case fully uses the approach laid out in the Generalized Minimum Rank Perturbation Theory section by exploring damage in both the mass and stiffness matrices simultaneously. When damage occurs in both matrices at the same time, the algorithm becomes iterative. This is due to the way the left eigenvectors are calculated. They need to be calculated from the unknown damaged mass or stiffness matrix as expressed in Eq. (12) and Eq. (13). An iterative process is carried out whereby the damage in the matrices is calculated using the healthy mass and stiffness matrices and then calculated with the updated mass and stiffness matrices until the process converges.

To determine the effectiveness of the algorithm a case where three linear and three nonlinear springs were damaged in the stiffness matrix, and the Coulomb friction increased in both locations was investigated using the exact eigenvalues and right eigenvectors of the system. The results illustrated in Fig. 5 show how the method predicts the exact damage in both the mass and stiffness matrices.

To determine the sensitivity of this method to measurement noise, a $3 \%$ random eigenvector perturbation and a $1 \%$ random eigenvalue perturbation was added to the case where three linear and three nonlinear springs were damaged in the stiffness matrix and the Coulomb friction increased in both locations. The results are illustrated in Fig. 6. The average damage values for 100 separate calculations were obtained and standard deviation error bars are plotted. The figure shows that the average value of the predicted damage is close to the exact damage (e.g. within $16 \%$ of the exact damage value in the augmented mass matrix).

The method was also explored by considering a $0.01 \%$ perturbation to the input of DSPI. The damage conditions were the same as in the previous scenario with three linear and three nonlinear springs damaged in addition to an increase in Coulomb friction in both locations. The results are shown in Fig. 7. Again the average value of the predicted damage is close to the

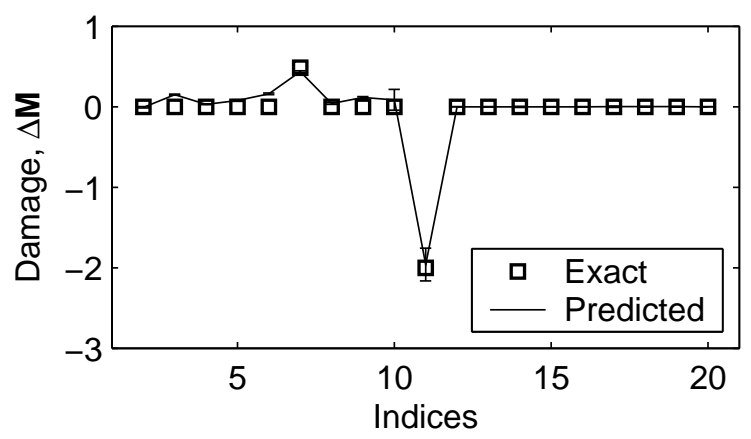

a) Damage in the mass matrix

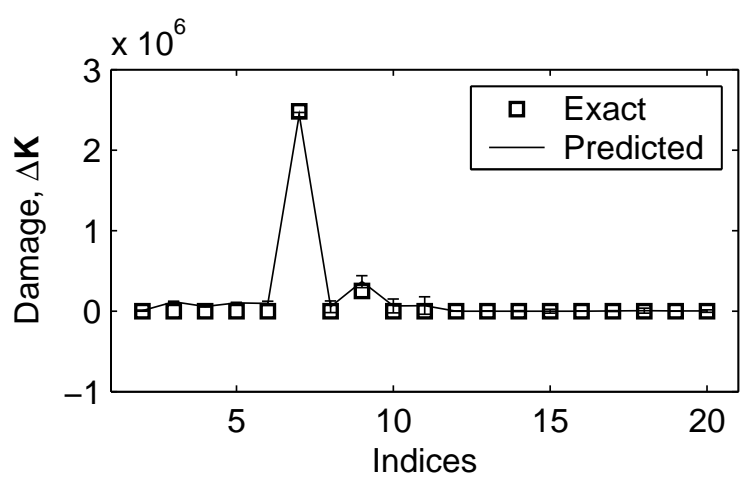

b) Damage in the stiffness matrix

Fig. 8 Case 3: Predicted damage in the nonlinear truss with damage associated with an increase in Coulomb friction (a), and a reduction of stiffness in a linear and a nonlinear spring (b) for a case with $3 \%$ random eigenvector noise and $1 \%$ random eigenvalue noise

exact damage (e.g. within $7 \%$ of the exact damage values in the augmented stiffness matrix).

\section{Case 3: Nonlinear Two Degree of Freedom Truss Structure}

This last case explores the application of the method to a more realistic structure. The approach was again carried out in the same iterative process that was explained in Case 2.

The sensitivity to modeling and measurement errors was again examined using $3 \%$ random eigenvector perturbation and $1 \%$ random eigenvalue perturbation to a scenario where a nonlinear spring was damaged, Coulomb friction increased at one of the locations, and simultaneously one of the beams had a decrease in stiffness. The results of the predicted damage for 100 separate calculations are shown in Fig. 8. The figure shows that the average value of the predicted damage is close to the exact damage (e.g. within $10 \%$ of the exact damage value in the augmented mass matrix).

\section{Conclusions}

A method to model nonlinear systems employing augmentation has been presented, and a damage detection method has been proposed. The proposed approach requires a discrete model for the system, and 
a known functional form of the nonlinearity. The nature of the augmentation requires the use of a modal analysis technique that has known (but not prescribed) forcing, such as DSPI. Once the eigenvalue problem is solved, the proposed approach predicts accurately both the location as well as the extent of damage.

A generalized minimal rank perturbation theory has been proposed to detect the location and extent of damage. This method is able to address the issue of asymmetric system matrices and asymmetric damage caused by nonlinearities (and the augmentation). For the case of simultaneous damage in the mass and stiffness matrices, the GMRPT algorithm becomes an iterative process.

The algorithms proposed have been demonstrated numerically for mass spring systems and a truss with cubic spring stiffnesses and Coulomb friction. The effectiveness of the proposed method has been demonstrated, and the effects of measurement errors have been discussed.

\section{Acknowledgments}

The authors wish to acknowledge the National Science Foundation (CAREER program) and Professor Masayoshi Tomizuka and Dr. Shih-Chi Liu (program directors) for the generous partial support of this work.

\section{References}

${ }^{1}$ M. S. Agbabian, S. F. Masri, R. F. Miller, and T. K. Caughey. System identification approach to detection of structural changes. ASCE Journal of Engineering Mechanics, 117(2):370-390, 1990.

${ }^{2}$ M. J. Atalla and D. J. Inman. On model updating using neural networks. Mechanical Systems and Signal Processing, 12(1):135-161, 1998.

${ }^{3}$ A. Bazoune, Y. A. Khulief, N. G. Stephen, and M. A. Mohiuddin. Dynamic response of spinning tapered Timoshenko beams using modal reduction. Finite Element Analysis and Design, 37(3):199-219, 2001.

${ }^{4}$ R. Bladh, M. P. Castanier, and C. Pierre. Reduced order modeling and vibration analysis of mistuned bladed disk assemblies with shrouds. Journal of Engineering for Gas Turbines and Power, 121(3):515-522, 1999.

${ }^{5}$ R. Bladh, M. P. Castanier, and C. Pierre. Componentmode-based reduced order modeling techniques for mistuned bladed disks - Part II: Application. Journal of Engineering for Gas Turbines and Power, 123(1):100-108, 2001.

${ }^{6}$ M. P. Castanier, Y. C. Tan, and C. Pierre. Characteristic constraint modes for component mode synthesis. AIAA Journal, 39(6):1183-1187, 2001.

${ }^{7}$ K. D'Souza and B. I. Epureanu. Damage detection in nonlinear systems using system augmentation and generalized minimum rank perturbation theory. Smart Materials and Structures, 2005. to appear.

${ }^{8}$ B. I. Epureanu, K. C. Hall, and E. H. Dowell. Reduced order models of unsteady transonic viscous flows in turbomachinery. Journal of Fluids and Structures, 14(8):1215-1235, 2000 .

${ }^{9}$ B. I. Epureanu, K. C. Hall, and E. H. Dowell. Reduced order models of unsteady viscous flows in turbomachinery using viscous-inviscid coupling. Journal of Fluids and Structures, 15(2):255-276, 2001.

${ }^{10}$ C. R. Farrar, S. W. Doebling, and D. A. Nix. Vibrationbased structural damage identification. Philosophical Trans- actions of the Royal Society of London: A - Mathematical, Physical and Engineering Sciences, 359(1778):131-149, 2001.

${ }^{11}$ B. F. Farrell and P. J. Ioannou. Accurate low-dimensional approximation of the linear dynamics of fluid flow. Journal of Atmosferic Sciences, 58(18):2771-2789, 2001.

${ }^{12}$ B. F. Feeny, C. M. Yuan, and J. P. Cusumano. Parametric identification of an experimental magneto-elastic oscillator. Journal of Sound and Vibration, 247(5):785-806, 2001.

${ }^{13} \mathrm{P}$. Feldmann and R. W. Freund. Efficient linear circuit analysis by Padé approximation via the lanczos process. IEEE Transactions on Computer Aided Design of Integrated Circuits and Systems, 14(5):639-349, 1995.

${ }^{14} \mathrm{~W}$. Heylen and P. Sas. Review of model optimization techniques. In Proceedings of the 5-th International Modal Analysis Conference, volume 2, pages 1172-1182, London, England, 1987.

${ }^{15} \mathrm{~S}$. R. Ibrahim and A. A. Saafan. Correlation of analysis in modeling and structures, assesment and review. In Proceedings of the 5-th International Modal Analysis Conference, volume 2, pages 1651-1660, London, England, 1987.

${ }^{16} \mathrm{M}$. Kamon, F. Wang, and J. White. Generating nearly optimally compact models from Krylov-subspace based reducedorder models. IEEE Transactions of Circuits and Systems II Analog and Digital Signal Processing, 47(4):239-248, 2000.

${ }^{17}$ M. Kaouk, D. C. Zimmerman, and T. W. Simmermacher. Assessment of damage affecting all structural properties using experimental modal parameters. ASME Journal of Vibration and Acoustics, 122(4):456-463, 2000.

${ }^{18}$ J. Leuridan. Some Direct Parameter Model Identification Methods Applicable For Multiple Modal Analysis. PhD thesis, University of Cincinnati, 1984.

${ }^{19}$ L. Ljung. System Identification - Theory for the User. Prentice Hall, New York, 1999.

${ }^{20}$ T. Marwala and H. E. M. Hunt. Fault identification using finite element models and neural networks. Mechanical Systems and Signal Processing, 13(3):475-490, 1999.

${ }^{21}$ S. F. Masri, R. K. Miller, A. F. Saud, and T. K. Caughey. Identification of nonlinear vibrating structures: Part I: Formulation. Journal of Applied Mechanics, 109(54):918-922, 1987.

${ }^{22}$ A. K. Pandey and M. Biswas. Damage detection in structures using changes in flexibility. Journal of Sound and Vibration, 169(1):3-17, 1994.

${ }^{23}$ A. W. Smyth, S. F. Masri, A. G. Chassiakos, and T. K. Caughey. On-line parametric identification of MDOF nonlinear hysteretic systems. ASCE Journal of Engineering Mechanics, 125(2):133-142, 1999.

${ }^{24}$ K. Worden and G. R. Tomlinson. Nonlinearity in experimental modal analysis. Philosophical Transactions of the Royal Society of London: A - Mathematical, Physical, and Engineering Sciences, 359(1778):113-130, 2001.

${ }^{25}$ Q. J. Yang, P. Q. Zhang, C. Q. Li, and X. P. Wu. A system theory approach to multi-input multi-output modal parameters identification methods. Mechanical Systems and Signal Processing, 8(2):159-174, 1994

${ }^{26} \mathrm{C}$. Zang and M. Imregun. Structural damage detection using artificial neural networks and measured FRF data reduced via principal component projection. Journal of Sound and Vibration, 242(5):813-827, 2001.

${ }^{27}$ D. C. Zimmerman. Looking into the crystal ball: The continued need for multiple viewpoints in damage detection. Key Engineering Materials: Damage Assessment of Structures, 167168(1):76-90, 1999.

${ }^{28}$ D. C. Zimmerman. Model validation and verification of large and complex space structures. Inverse Problems in Engineering, 8(2):93-118, 2000

${ }^{29}$ D. C. Zimmerman and M. Kaouk. Structural damage detection using minimum rank update theory. ASME Journal of Vibration and Acoustics, 116(2):222-231, 1994.

${ }^{30}$ D. C. Zimmerman and T. Simmermacher. Model correlation using multiple static load and vibration tests. AIAA Journal, 33(11):2182-2188, 1995. 